\title{
MEASUREMENT OF THE ANALYZING POWER IN PP ELASTIC SCATTERING IN THE PEAK CNI REGION AT RHIC
}

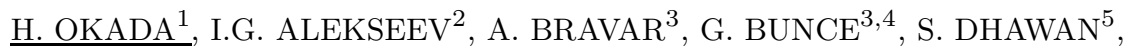 \\ R. GILL ${ }^{3}$, W. HAEBERLI ${ }^{6}$, O. JINNOUCHI ${ }^{4}$, A. KHODINOV $^{7}$,

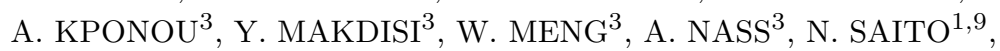 \\ H. SPINKA ${ }^{10}$, E.J. STEPHENSON ${ }^{11}$, D.N. SVIRIDA ${ }^{2}$, T. WISE ${ }^{6}$, \\ A. ZELENSKI ${ }^{3}$ \\ ${ }^{1}$ Kyoto Univ. ${ }^{2}$ ITEP ${ }^{3}$ BNL ${ }^{4}$ RIKEN BNL Research Center ${ }^{5}$ Yale \\ Univ. ${ }^{6}$ Univ. of Wisconsin-Madison ${ }^{7}$ Stony Brook \\ Univ. ${ }^{8}$ RIKEN ${ }^{9}$ ANL ${ }^{10}$ Indiana Univ. \\ E-mail: okadah@bnl.gov
}

\begin{abstract}
The analyzing power $A_{N}$ for pp elastic scattering is expected to reach a peak value of 0.045 in the Coulomb Nuclear Interference (CNI) region at a momentum transfer $-t$ of $0.003(\mathrm{GeV} / c)^{2}$. During the 2004 RHIC Run, we completed a measurement of $A_{N}$ in the CNI region by detecting the recoil protons from $p p$ elastic scattering using a polarized atomic hydrogen gas jet target and the 100 $\mathrm{GeV}$ RHIC proton beam. We report the first measurements of the $A_{N}$ absolute value and shape in the $-t$ range from 0.0015 to $0.010(\mathrm{GeV} / c)^{2}$ with a precision better than 0.005 for each $A_{N}$ data point. The recoil protons were detected with two arrays of $\mathrm{Si}$ detectors. The absolute target polarization as monitored by a Breit-Rabi polarimeter was stable at $0.924 \pm 0.018$. This result allows us to further investigate the spin dependence of elastic pp scattering in the very low $-t$ region.
\end{abstract}

The elastic scattering of nucleons has been studied for a long time as the most fundamental reaction to extract information on the elementary building blocks of matter, nucleons. Regarding the initial and final states in elastic scattering of protons, there are three types of processes ; no spin reversal, single spin reversal and double spin reversal processes. These processes can be described by the use of five independent helicity amplitudes which have hadronic and electromagnetic parts. The electromagnetic part of the helicity amplitudes is well understood by QED. On the other hand, the hadronic part is being studied ${ }^{1}$. The analyzing power $\left(A_{N}\right)$ mainly depends on the single spin reversal process. At very small momentum transfer $-t=0.001$ to $0.02(\mathrm{GeV} / c)^{2}$, two helicity amplitudes (hadronic 
and electro magnetic) become similar in size and interfere with each other. We call this phenomenon Coulomb Nuclear Interference (CNI). The values of $A_{N}$ is expected to reach a peak of 0.045 at $-t \sim 0.003(\mathrm{GeV} / c)^{2}$. The shape of $A_{N}$ is related to the hadronic amplitude of the single spin reversal process. Experimetal knowledge is needed to constrain theory. The only $A_{N}$ data available in the CNI region is from E704 at Fermilab ${ }^{2}$ with limited accuracy. Last spring (the 2004 RHIC run), we carried out a measurement of $A_{N}$ in the peak CNI region using a polarized atomic hydrogen gas jet target.

Since the CNI process is ideal to be used as a polarimeter for a high energy proton beam ${ }^{3}$ and to determine the RHIC beam polarization, we have installed the RHIC Absolute Polarimeter ${ }^{4}$. This polarimeter consists of the silicon spectrometer and the polarized atomic hydrogen-jet target, which includes a Breit-Rabi polarimeter to obtain a precise absolute target polarization ${ }^{5}$. By knowing the absolute polarization of the target $\left(P_{\text {target }}\right)$, we measured the absolute $A_{N}$ from the scattering asymmetry with respect to the target spin sign $\left(\epsilon_{\text {target }}\right)$, that is, $A_{N}=\epsilon_{\text {target }} / P_{\text {target }}$. Since we are using the elastic scattering process of proton beam and proton target, we also obtain the absolute beam polarization from the scattering asymmetry with respect to the beam and $A_{N}$. Figure 1 shows the schematic of the setup. We detect the recoil particles by using 3 left-right pairs of silicon strip detectors. The strips run vertically with respect to the beam direction. The read out pitch is about $4 \mathrm{~mm}$ and each channel outputs energy $\left(T_{R}\right)$ and time of flight (tof). The readout channel number gives us the angle $\left(\theta_{R}\right)$ information as shown in Fig. 2.

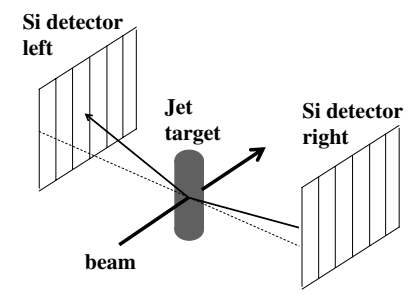

Figure 1. Setup

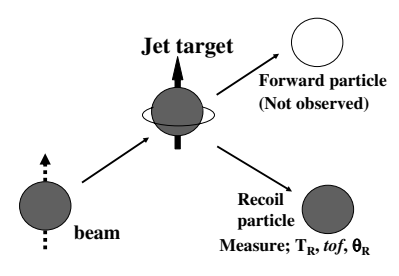

Figure 2. Elastic scattering process 
To get $A_{N}$, we measure the left-right asymmetry of the elastic event yield with respect the target spin sign, then divide it by target polarization. The key points of this experiment are high target polarization and the capability of selecting the elastic events. The target polarization was quite high, accurate $\left(P_{\text {target }}=0.924 \pm 0.018\right)$ and stable $^{5}$. We confirmed that both the recoil particle mass and the forward-scattered particle mass (missing mass) were consistent with that of the proton using the following two correlations. $T_{R}$ and tof confirm the recoil particle is proton (Fig. 3). $T_{R}$ and channel number $\left(\propto \theta_{R}\right)$ confirm the forward scattered particle is proton (Fig. 4). We have focused on the smaller $T_{R}$ region $(<5 \mathrm{MeV})$ which corresponds to $-t$ region of $0.0015<-t<0.01(\mathrm{GeV} / \mathrm{c})^{2}$.

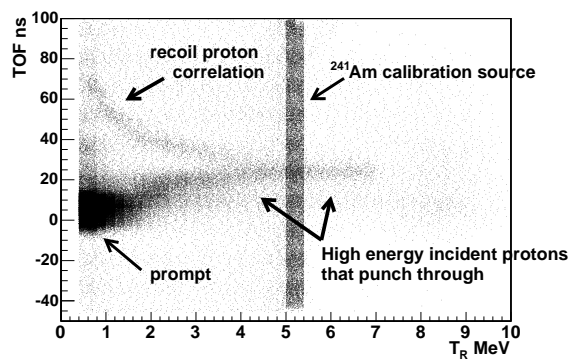

Figure 3. The correlation of $T_{R}$ and tof of one detector. This confirms the recoil particle is a proton.

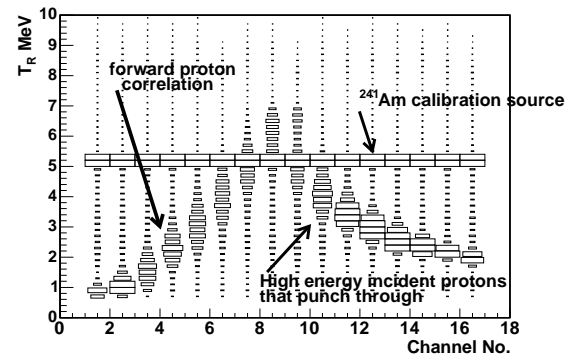

Figure 4. The correlation of $T_{R}$ and channel number $\left(\propto \theta_{R}\right)$ of one detector. Each detector has 16 channels. This confirms the forward scattered particle is a proton (less than $5 \mathrm{MeV}$ ). Because the higher energy protons punched through the silicon detector, the measured $T_{R}$ decreases beyond channel number 9 . 
Figure 5 shows our result (closed circles) and E704 data (open circles). The errors are statistical only. We estimate the systematic error in $A_{N}$ to be 0.0015 , which is dominated by possible contributions of background (beam gas interaction etc.). The fraction of background is less than $5 \%$.

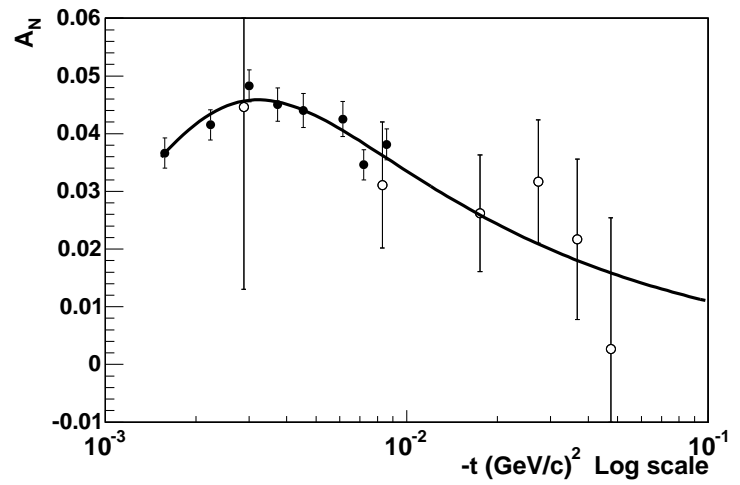

Figure 5. $A_{N}$ data for $p p$ elastic scattering as a function of $-t$. The closed 8 circles are the result; the open circles are from E704, The errors shown are statistical only. The solid line is a no hadronic spin flip calculation. $\chi^{2} /$ d.o.f. $=6 / 8$, which includs statistical and systematic errors.

We have measured $A_{N}$ in the peak CNI region from 0.0015 to 0.010 $(\mathrm{GeV} / c)^{2}$ in $-t$, and observed the peak shape of $A_{N}$ for the first time.

The authors would like to thank the BNL Instrumental Division for their work on the silicon detectors and electronics. The work is performed under the auspices of U.S. DOE contract Nos. DE-AC02-98CH10886 and W31-109-ENG-38, DOE grant No. DE-FG02-88ER40438, NSF grant PHY0100348, and with support from RIKEN, Japan.

\section{References}

1. T.L. Trueman, these proceedings (2004).

2. N. Akchurin et al.,Phys. Rev. D 48, 3026 (1993).

3. O. Jinnouchi et al., these proceedings (2004) and AIP Conf. Proc. 675 (2003) 817.

4. A. Bravar et al., these proceedings (2004) and AIP Conf. Proc. 675 (2003) 830.

5. A. Zelenski, T. Wise and A. Nass et al., these proceedings (2004). 